\title{
Neonatal outcome of maternal premature rupture of membranes more than 18 hours
} Shakya A ${ }^{1}$ iD, Gupta SK²

${ }^{1}$ Amshu Shakya, Registrar (Pediatric Cardiology Unit), Shahid Gangalal National Heart Centre, Bansbari, Kathmandu, Nepal; ${ }^{2}$ Sweta Kumari Gupta, Consultant Pediatrician, Bharatpur Hospital, Chitwan, Nepal.

\begin{abstract}
Background: Premature Rupture of Membranes has been known to complicate pregnancy since ages. Several risk factors may be associated with occurrence of premature rupture of membranes. It is associated with varied neonatal complications, neonatal sepsis and prematurity being the most hazardous ones.

Objectives: This study was conducted to evaluate the incidence of neonatal sepsis following premature rupture of membranes, risk factors and neonatal complications associated with premature rupture of membrane of more than 18 hours in the neonates admitted in a tertiary care center.

Methodology: Descriptive observational study was undertaken for 18 months from August 2013 to January 2015 in the College of Medical Sciences, Chitwan, Nepal. This study included 82 neonates admitted to the Neonatal Intensive Care Unit with maternal history of premature rupture of membranes of more than 18 hours with medical conditions excluded. Results: The incidence of premature rupture of membranes in this hospital was $8.9 \%$. Frequently associated maternal risk factors were history of prior abortion (16, 19.5\%), urinary tract infection $(7,8.5 \%)$, and antecedent coitus $(7,8.5 \%)$. In the study population, 68 (83\%) neonates had complications and 14 (17\%) neonates had no complication. The incidence of neonatal sepsis following PROM in present study was 6.1\%. Neonatal sepsis was significantly associated with chorioamnionitis $(\mathrm{p}<0.001)$. The most frequently occurring complication was probable neonatal sepsis $(53,64.6 \%)$, followed by prematurity $(31,37.8 \%)$ and perinatal asphyxia $(15,18.3 \%)$. There was a directly proportional relation of neonatal sepsis, chorioamnionitis, respiratory distress syndrome and mortality with duration of premature rupture of membranes.

Conclusion: Commonly occurring maternal risk factors with PROM were prior abortion, UTI and antecedent coitus. In the study population, infectious morbidity was highest. Chorioamnionitis was significantly associated with culture proven neonatal sepsis. Prolonged duration of premature rupture of membranes increased the risk of neonatal sepsis, meningitis, respiratory distress syndrome and perinatal death. Prevention of these risk factors, appropriate and timely management including improved obstetric and neonatal care can provide intact survival of the neonates.
\end{abstract}

Key words: Chorioamnionitis; Neonatal sepsis; Premature rupture of membranes

Access this article online

Website: www.jkmc.com.np

DOI: https://doi.org/10.3126/jkmc.v9i2.35526

HOW TO CITE

Shakya A, Gupta SK.Neonatal outcome of maternal premature rupture of membranes. J Kathmandu Med Coll. 2020;9(2):81-6.

Address for correspondence

Dr. Amshu Shakya

Registrar (Pediatric Cardiology Unit)

Shahid Gangalal National Heart Centre, Bansbari, Kathmandu, Nepal

E-mail: amshushakya@hotmail.com

ORCID: https://orcid.org/0000-0002-1770-494X

Copyright @ 2020 Journal of Kathmandu Medical College (JKMC)

ISSN: 2019-1785 (Print), 2091-1793 (Online)

\section{INTRODUCTION}

Dremature rupture of membranes (PROM) occurs in approximately $10 \%$ of all pregnancies. When this event occurs before 37 weeks of gestation, it is termed as preterm premature rupture of membranes (PPROM) that has been estimated to affect $3 \%$ to $4.5 \%$ of all deliveries'. The time between the rupture of membranes and the onset of labor is defined as the latency period. Thus, premature rupture of membranes affect both preterm pregnancies to term pregnancies, and childbirth may be premature or not, depending on how long the period of latency is ${ }^{2}$. 
When this event lasts more than 18 hours before labor, it is regarded as prolonged, which is associated with an increased rate of neonatal infections to relatively ten folds $s^{3}$.

Rupture of membranes often leads to the onset of labor. In term pregnancy, rupture of membranes leads to spontaneous labor in $70 \%$ cases within 24 hours $^{4}$. And delivery occurs within 7 days of PPROM in over $80 \%$ of cases $^{5}$.

The etiology of PROM seems to be multifactorial ${ }^{3}$. The complications of PROM for the infant are preterm delivery, RDS, infection (Pneumonia, meningitis, sepsis), pulmonary hypoplasia, limb and body deformities, umbilical cord compression, abruption and cord prolapse ${ }^{6}$.

There is paucity of studies regarding PROM and its implications in neonates in Nepal. This research was conducted to help health professionals identify maternal risk factors and neonatal outcomes following prolonged PROM, so that health professionals who assist them will be able to deliver a more targeted assistance thereby helping to reduce the incidence of newborns' morbidities.

\section{METHODOLOGY}

Patients: All live born neonates with maternal history of premature rupture of membranes more than 18 hours. Babies born to mothers with toxemia of pregnancy, Medical disease other than infection were excluded from the study.

During this study period the standard management was as follows:

Obstetric practice: The documentation of the rupture of membranes was done by sterile speculum examination confirming the pooling of amniotic fluid in the posterior vaginal fornix or/and direct visualization of fluid leakage from the cervical canal by the Gynecology/ Obstetric Department of COMS-TH. The diagnosis of chorioamnionitis was made clinically. Presence of fever $38^{\circ} \mathrm{C}$ and at least 2 of the following conditions were regarded as Chorioamnionitis ${ }^{6}$,

i) Maternal tachycardia $>100 / \mathrm{min}$,

ii) Fetal tachycardia $>160 / \mathrm{min}$,

iii) Uterine tenderness,

iv) Foul smelling amniotic fluid

v) Maternal leukocytosis $>15000 / \mathrm{mm} 3$

vi) $\mathrm{CRP}>2.7 \mathrm{mg} / \mathrm{dl}$

vii) With no other site of infection.
Mothers with suspicious chorioamnionitis were given antibiotics immediately after admission. In neonates delivered outside, rupture of membranes was diagnosed based on maternal history of passage of gush of fluid per vaginum before onset of labor pain. Neonatal sepsis was diagnosed when generalized systemic features were associated with pure growth of bacteria in one or more sites and Neonatal sepsis was presumed when clinical and laboratory findings are consistent with bacterial infection but blood culture was sterile ${ }^{7}$. Meningitis was diagnosed based on history, clinical examination, septic screen, blood culture and analysis of the cerebrospinal fluid for cell count, protein, glucose concentration, gram stain and culture ${ }^{8}$. History and clinical evaluation consistent with hyaline membrane disease and typical chest $\mathrm{x}$-ray findings in a preterm baby with no other alternative diagnosis like congenital pneumonia, was diagnosed as respiratory distress syndrome'. Slow gasping or no breathing at birth or Apgar score less than 4 at 5 minutes was regarded as perinatal asphyxia ${ }^{10}$. Birth asphyxia was presumed in home deliveries when there was a history that the baby had failed to cry or breathe at birth, had gasped for a long time, had to be stimulated for a prolonged period of time, or was unable to suck in the first 24 hours.

Neonatal practice: During the study period, 1666 deliveries were conducted in the hospital. 154(149 born in our hospital and 5 referred from other hospitals) neonates had history of PROM, 21 refused admission, 41 had associated maternal exclusion criteria and 10 left against medical advice, so study was completed in 82 neonates.

These neonates were admitted in the hospital, septic screening sent, and antibiotics started (Injection Cefotaxime and Injection Amikacin). Antibiotics were discontinued after 72 hours if the baby did not have any symptoms and blood cultures were sterile. Urine and CSF culture were only done in cases presenting after 72 hours of life or those with positive blood culture reports. Antibiotics were tailored according to culture sensitivity reports and the duration of antibiotics depended upon the underlying condition and clinical improvement.

This Hospital based descriptive observational study was conducted for 18 months from August 2013 to January 2015 in the Department of Pediatrics (Neonatal Intensive Care Unit) and Post natal ward of College of Medical Sciences, Bharatpur. Subjects were thoroughly evaluated by the investigator as per designed proforma which included history, physical examination, and course during hospitalization. Study variables were maternal 
risk factors and neonatal complications. Analysis was performed using SPSS (Statistical Package for Social Sciences) version 20. Statistical method included determination of $\mathrm{p}$-value using $\mathrm{Chi}$ - square test. For tables where expected frequencies were less than five, Fisher's Exact test was performed. Statistical test was considered significant at 0.05 level of confidence.

\section{RESULTS}

The most common maternal age group was 20-35 years, $77(89 \%)$. The majority of mothers were primigravida, 47 (57.3\%).36 (43.9\%) mothers belonged to lower middle class families. Most mothers were housewives, 53 (64.6\%).47 (57.3\%) mothers had up to secondary level of education.

The incidence of PROM was $8.9 \%$.

Maternal risk factors: Commonly occurring risk factors were prior history of abortions [16 (19.5\%)], antecedent coitus [7 (8.5\%)] and urinary tract infections [7 (8.5\%)].

Neonatal complications: In the study population, 68 (83\%) neonates had complications and 14 (17\%) neonates had no complication. The incidence of neonatal sepsis among the study population was $6.1 \%$. Maternal chorioamnionitis was significantly associated with neonatal sepsis $(p<0.001)$. Most common complication was probable neonatal sepsis [53 (64.6\%)] followed by hyperbilirubinemia [35 (42.7\%)], prematurity [31 (37.8\%)], perinatal asphyxia [15 (15.9\%)], IUGR [6 (7.3\%)], neonatal sepsis [5 (6.1\%)], RDS [3 (3.7\%)], congenital anomalies [2 (2.4\%)], meningitis [2 (2.4\%)] and perinatal death [1 (1.2\%)]. The statistically significant association was seen with probable neonatal sepsis among terms $(p=0.044)$ and RDS in preterm neonates $(p=0.021)$. There was significant association between oligohydramnios and congenital anomalies ( $p$-value $=0.023$ ). Neonatal sepsis, meningitis, RDS, chorioamnionitis and perinatal death increased with increasing duration of PROM.

Table 1: Maternal socio-demographic characteristics

\begin{tabular}{llc}
\hline Variables & Subgroup & No of patients(\%) \\
\hline Age & $<20$ & $7(8.5)$ \\
& $20-35$ & $73(89)$ \\
Gravida & $>35$ & $2(2.5)$ \\
& Primi & $47(57.3)$ \\
Level of education & Multi & $35(42.7)$ \\
& Illiterate & $4(4.9)$ \\
& Primary level & $13(15.9)$ \\
Socioeconomic status & Secondary level & $47(57.3)$ \\
(Kuppuswamy) ${ }^{11}$ & Undergraduate and higher & $18(21.9)$ \\
& Upper class & $2(2.4)$ \\
& Middle upper & $30(36.6)$ \\
Employment & Lower middle & $36(43.9)$ \\
& Upper lower & $14(17.1)$ \\
& Lower & $0(0)$ \\
\hline
\end{tabular}

Table 2: Distribution of neonates according to maternal risk factors

\begin{tabular}{lc}
\hline Variables & No of patients(\%) \\
\hline Multiple gestation & $2(2.4)$ \\
\hline CPD & $3(3.2)$ \\
UTI & $7(8.5)$ \\
\hline Abnormal PV discharge & $1(1.2)$ \\
\hline Previous abortion & $16(19.5)$ \\
\hline Previous preterm birth & $2(2.4)$ \\
\hline Polyhydramnios & $1(1.2)$ \\
\hline Oligohydramnios & $2(2.4)$ \\
\hline Smoking & $2(2.4)$ \\
\hline Antecedent coitus & $7(8.5)$ \\
\hline
\end{tabular}


Table 3: Distribution of Neonates according to complications

\begin{tabular}{llc}
\hline Complication & Subgroups & No. of patients (\%) \\
Neonatal sepsis & \multicolumn{1}{c}{$5(6.1)$} \\
\hline Probable neonatal sepsis & Chorioamnionitis & $3(60)$ p $<0.001$ \\
\hline Meningitis & & $53(64.6)$ \\
\hline Prematurity & $28-31$ weeks & $2(2.4)$ \\
& $32-37$ weeks & $4(4.9)$ \\
\hline Respiratory distress syndrome & $>37$ weeks & $27(32.9)$ \\
Perinatal Asphyxia & & $31(62.2)$ \\
\hline Hyperbilirubinemia & & $3(3.7)$ \\
IUGR & & $15(18.3)$ \\
\hline Congenital anomalies & $35(42.7)$ \\
\hline Perinatal mortality & $6(7.3)$ \\
\hline
\end{tabular}

Table 4: Distribution of neonatal complication as per gestational age

\begin{tabular}{|c|c|c|c|c|}
\hline \multirow{2}{*}{ Neonatal morbidities } & \multicolumn{3}{|c|}{ No. of patients in different Gestational age in weeks } & \multirow{2}{*}{ p-value } \\
\hline & 28-31 weeks & 32-37 weeks & $>37$ weeks & \\
\hline RDS (Yes) & $1(33.3)$ & $2(66.7)$ & $0(0)$ & 0.021 \\
\hline Neonatal sepsis (Yes) & $0(0)$ & $3(60)$ & $2(40)$ & 0.485 \\
\hline Probable neonatal sepsis (Yes) & $4(7.5)$ & $21(39.6)$ & $28(52.8)$ & 0.044 \\
\hline Meningitis (Yes) & $0(0)$ & $0(0)$ & $2(100)$ & 0.585 \\
\hline Perinatal asphyxia (Yes) & $0(0)$ & $4(26.7)$ & $11(73.3)$ & 0.618 \\
\hline Intrauterine growth restriction (Yes) & $0(0)$ & $0(0)$ & $6(100)$ & 0.170 \\
\hline Hyperbilirubinemia (Yes) & $4(11.4)$ & $19(54.3)$ & $12(34.2)$ & $<0.001$ \\
\hline Congenital anomalies (Yes) & $0(0)$ & $2(100)$ & $0(0)$ & 0.201 \\
\hline Perinatal mortality (Yes) & $0(0)$ & $0(0)$ & $1(100)$ & 1.000 \\
\hline
\end{tabular}

Table 6: Distribution of neonatal complication as per duration of PROM

\begin{tabular}{|c|c|c|c|c|}
\hline \multirow{2}{*}{ Neonatal morbidities } & \multicolumn{3}{|c|}{ Duration of PROM } & \multirow[b]{2}{*}{ p-value } \\
\hline & 18-24 hours & 24-48 hours & $>48$ hours & \\
\hline RDS (Yes) & $0(0)$ & $0(0)$ & $3(100)$ & 0.056 \\
\hline Neonatal sepsis (Yes) & $1(20)$ & $1(20)$ & $3(60)$ & 0.513 \\
\hline Probable neonatal sepsis (Yes) & $18(34.0)$ & $16(30.2)$ & $19(35.8)$ & 0.044 \\
\hline Meningitis (Yes) & $0(0)$ & $1(50)$ & $1(50)$ & 0.524 \\
\hline Perinatal asphyxia (Yes) & $8(53.3)$ & $1(6.7)$ & $6(40)$ & 0.092 \\
\hline Perinatal mortality (Yes) & $0(0)$ & $0(0)$ & $1(100)$ & 0.622 \\
\hline
\end{tabular}

\section{DISCUSSION}

Incidence of PROM in this study was $8.9 \%$ which is comparable to other studies. The incidence of PROM was $7.7 \%$ and $6.9 \%$ in studies done by Boskabadi et al. $(2011)^{3}$ and Nili et al. $(2003)^{1}$ respectively. The most frequently associated maternal risk factors were history of prior abortion (16, 19.5\%), urinary tract infection (7, 8.5\%), and antecedent coitus $(7,8.5 \%)$.It is in concordance with study by Patil et al. (2014) ${ }^{12}$ where the percentage of women with PROM with history of recent coitus was $10 \%$ and UTI was $6 \%$. Theoretically, sexual intercourse and UTI may increase the possibility of ascending infection thus leading to $\mathrm{PROM}^{12}$. A study done at Punjab Medical College, Faisalabad, Pakistan showed $17.6 \%$ of mothers with PROM had a history of previous abortion ${ }^{13}$. Repeated abortions which are managed with dilatation 
and evacuation damage the function of cervix musculi sphincter lead to dilated cervix. And dilated cervix can cause PROM in successive pregnancies ${ }^{14}$.

The incidence of neonatal sepsis following PROM in present study was $6.1 \%$. Neonatal sepsis was significantly associated with chorioamnionitis $(p<0.001)$.This is in concordance with results given by Asindi et al. (2002) ${ }^{15}$ where chorioamnionitis was present in upto $50 \%$ among the infants born to mothers with PROM with proven sepsis but only in $3.3 \%$ among infants with no proven sepsis. Alam et al. (2014) ${ }^{16}$ also emphasized on the importance of chorioamnionitis ( $<<0.001 ; A O R, 4.1)$ as an independent risk factor for culture proven neonatal sepsis in PROM in their study.

Prolonged duration of PROM increased the risk of neonatal sepsis, RDS and perinatal death. It is consistent with study by Alam et al. (2014) ${ }^{16}$, they reported that yield of culture positivity was eight times more in neonates with more than 48 hours of maternal PROM at the time of birth. Culture negative Meningitis in this study occurred in neonates with mother with PROM of more than 24 hours. No one developed meningitis in neonates of mothers with PROM of 18-24 hours. It hints that prolonged duration of PROM has some bearing over

\section{REFERENCES}

1. F. Nili AA. Shams Ansari. Neonatal Complications of Premature Rupture of Membranes. Acta Med Iran. 41(3):175-179. [FullText]

2. Silviera ML, De Oliviera Caminha N, De Sousa RA, Maria Fraxe Pessoa S, De Paula Pessoa Gurgel E, Maria Prudêncio Cavalcante D. Neonatal outcome in pregnancies that presented premature rupture of membranes. Rev Rene 2014;15(3):491-8. [DOI]

3. Boskabadi H, Maamouri G, Mafinejad S. Neonatal Complications Related with Prolonged Rupture of Membranes. Macedonian Journal of Medical Sciences 2011;4(1):93-8. [DOI]

4. Shrestha SR, Sharma PN. Fetal Outcome of prelabour rupture of membranes. J. Obstet. Gynaecol. 2006;2:19-24. [DOI]

5. Kornacki J, Goździewicz T, Łabędzka I, Gruca-Stryjak K, Kornacka A, Skrzypczak J, et al. The influence of preterm premature rupture of membranes on maternal and neonatal outcome. Archives of Medical Science. 2009;5(2):222-8. [FullText]

6. Premature rupture of membranes. In: Arias F, Daftary SN, Bhide AG, editors. Practical guide to High-Risk Pregnancy and Delivery: A South Asian Perspective. 3rd ed. New Delhi: Elsevier; 2008. p. 240-61. the occurrence of meningitis in neonates. Infectious neonatal morbidities increased with prolonged duration of $\mathrm{PROM}^{2}$.

RDS was major neonatal morbidity in neonates with PPROM like in studies by Khanal et al. (2009) ${ }^{17}$ and Boskabadi et al. $(2011)^{3}$, they also mentioned that duration of PROM increased the risks of RDS. Rauf et al. $(2014)^{18}$ proposed a proportional relation between duration of PROM and perinatal asphyxia.

This study is limited by lack of long term follow up of patients born to mothers with premature rupture of membrane for more than 18 hours. Also being a single centre study with limited number of patients, the results should be generalized with caution.

\section{CONCLUSION}

This study helps to clarify the risk factors and probable complications of Premature rupture of membranes. Appropriate measures should be taken to reduce abortions, UTI and antecedent coitus to prevent PROM. Neonates born to mothers with PROM of more than 18 hours should be observed vigilantly for neonatal sepsis, RDS or perinatal asphyxia as well as other morbidities.

7. Singh M. Perinatal infections. In: Singh M. Care of the newborn. 7th ed. New Delhi: Sagar Publications; 2010. p. 208-33.

8. Puopolo KM. Bacterial and fungal infections. In: Cloherty JP, Eichenwald EC, Stark AR, Hansen AR, editors. Manual of Neonatal Care. 7th ed. Philadelphia: Lippincott Williams \& Wilkins; 2008. p. 624-55.

9. Bhakta KY. Respiratory distress syndrome. In: Cloherty JP, Eichenwald EC, Hansen AR, Stark AR, editors. Manual of Neonatal Care. 7th ed. New Delhi: Lippincott Williams \& Wilkins; 2012. p. 406-7.

10. Introduction to care of newborn babies. In: Singh M. Care of the newborn, 7th ed. New Delhi: Sagar Publications; 2010. p. 1-12.

11. Ghosh A, Ghosh T. Modified Kuppuswamy's Socioeconomic Status Scale in the context of Nepal. Indian Pediatr. 2009;46:1104-5. [FullText]

12. Patil S, Patil V. Maternal and Foetal Outcome in Premature Rupture of Membranes. IOSR JDMS. 2014;13(12):56-83. [DOI]

13. Tahir S, Aleem M, Aziz R. Incidence and outcome of preterm premature rupture of membranes. Pakistan Journal of Medical and Health Sciences. 2002;18(1):26-32. 
14. Li N, Fu Q, Cai W. Cause analysis and clinical management experience of thepremature rupture of membrane. Open Journal of Obstetrics and Gynecology. 2013;3:222-6.[DOI]

15. Asindi AA, Archibong El, Mannan NB. Mother-infant colonization and neonatal sepsis in prelabor rupture of membranes. Saudi Med J. 2002;23(10):1270-4. [PubMed]

16. Alam MM, Salim AF, Shaikh AS, Munir O, Qadir M. Neonatal sepsis following prolonged rupture of membranes in a tertiary care hospital in Karachi,
Pakistan. J Infect Dev Ctries. 2014;8(1):067-73. [PubMed] [DOI]

17. Khanal S, Zhang W, Shrestha N R, Dahal GR. A comparative study of outcome of preterm neonate with and without history of preterm premature rupture of membrane. Nepal Med Coll J. 2009;11(2):99-103. [PubMed]

18. Rauf A, Mehta S, Beniwal NS. Maternal \& Foetal Outcome in Cases of Pre-Labour Rupture of Membranes. IORS-JDMS 2014;13(11):8-11. [DOI] 